\title{
Synergic Effects of Cranial Electrotherapy Stimulation with Sleep Hygiene in Patients with Chronic Insomnia
}

\author{
Dong Rak Kwon ${ }^{1}$, Jung A Park ${ }^{2}$, Young Soo Lee ${ }^{3}$, Jae Hyuk Kwak ${ }^{2}$, Jin Kuk Do ${ }^{2 *}$, Ji Eun Kim ${ }^{2 *}$ \\ ${ }^{1}$ Departments of Rehabilitation Medicine and ${ }^{2}$ Neurology, Daegu Catholic University School of Medicine, Daegu, \\ ${ }^{3}$ Division of Cardiology, Department of Internal Medicine, Daegu Catholic University School of Medicine, Daegu, Korea
}

Received May 19, 2019

Revised June 16, 2019

Accepted June 19, 2019

Address for correspondence

Ji Eun Kim, MD, PhD

Department of Neurology,

Daegu Catholic University

School of Medicine,

33 Duryugongwon-ro 17-gil,

Nam-gu, Daegu 42472, Korea

Tel: +82-53-650-4268

Fax: +82-53-654-9786

E-mail: jekim2@cu.ac.kr

Jin Kuk Do, MD, PhD

Department of Neurology,

Daegu Catholic University

School of Medicine,

33 Duryugongwon-ro 17-gil,

Nam-gu, Daegu 42472, Korea

Tel: $+82-53-650-4269$

Fax: +82-53-654-9786

E-mail: neuropia@cu.ac.kr
Objectives: To investigate the therapeutic effect of cranial electrotherapy stimulation (CES) with sleep hygiene in patients with chronic insomnia. Methods: This study was designed as a prospective, doubleblinded, and randomized controlled trial. Twenty-seven patients with chronic insomnia were recruited and randomly allocated to two groups; cranial microcurrent therapy (MC) group and sham group. All patients received sleep hygiene education. Pittsburgh Sleep Quality Index (PSQI) and Insomnia Severity Index (ISI) were measured at baseline (pre-treatment), and 2 weeks and 4 weeks of treatment. Results: In MC group, the PSQI and ISI showed a tendency to decrease consistently until 4 weeks of treatment. In sham group, PSQI and ISI initially decreased during the first 2 weeks, but it increased after 2 weeks of treatment. Conclusions: This study showed that combination treatment of CES and sleep hygiene is more effective in treating chronic insomnia than sleep hygiene only as demonstrated by improvement and maintenance of sleep score for 1 month.

J Sleep Med 2019;16(1):36-40

\section{Introduction}

Chronic insomnia is a crucial problem which affects the quality of life and health status. It is no longer just an extension of psychiatric disease in that increasing evidence supports that chronic insomnia is associated with medical problems such as increased cardiovascular risks. ${ }^{1,2}$ Cognitive behavioral therapy for insomnia (CBT-I) and pharmacotherapy are the main treatment option for the chronic insomnia. The CBT-I is usually recommended and preferred as first-line therapy due to its low side effects and better outcomes over pharmacotherapy. ${ }^{3}$ Despite the efforts to treat the disorder, chronic insomnia is still considered to be a persistent condition due to its variable remission rates and high recurrence rates even after remission. ${ }^{4,5}$

This is an Open Access article distributed under the terms of the Creative Commons Attribution Non-Commercial License (https://creativecommons.org/licenses/by-nc/4.0) which permits unrestricted non-commercial use, distribution, and reproduction in any medium, provided the original work is properly cited.
Cranial electrotherapy stimulation (CES) is a novel attempt to treat the chronic insomnia. It is one of the methods of brain stimulation using low-intensity electrical currents. ${ }^{6}$ Usually, the electrodes are placed at the structures of the head, such as earlobes, mastoids, or temples. The low-voltage microcurrents less than or near $2 \mathrm{~mA}$ are given through the electrodes. There are two major forms in the CES: transcranial direct current stimulation (tDCS) and cranial alternating current (AC) stimulation. According to recent studies about the mechanism of CES, tDCS is known to change the cortical excitability by its polarizing effects on the brain tissue. The AC stimulation is believed to make its effects on the brain by changing the electrochemical responses of the synapse and neural networks. ${ }^{7}$ Both methods are expected to modulate the brain activity, especially the psychophysiological status including chronic insomnia.

Many studies adopted CES for the treatment of depression, anxiety, chronic pain and insomnia. ${ }^{8}$ The early studies in $1900 \mathrm{~s}$ 
found that CES induced the subject to sleepy state which made it initially called "electrosleep," or "electronarcosis." Gilula and Kirsh, in 2005, found that CES was proved to be efficacious for relieving the symptom of depression in $81 \%$ of the previous studies. ${ }^{10}$ Our study was aimed to investigate the therapeutic effect of cranial microcurrent therapy (MC) with sleep hygiene in patients with chronic insomnia.

\section{Methods}

\section{Study design}

This study used a prospective, randomized, double blinded, sham-controlled design and was performed at Daegu Catholic University Medical Center (DCUMC), Daegu, South Korea, from December 2016 to October 2017. The study was approved by the Institutional Review Board (IRB, MDCR16-013) of DCUMC. Written informed consent was obtained from each participant. There were 34 patients initially screened and enrolled for the study. But 7 patients were finally excluded because they refused to participate in the trial in their second visit. Finally, twenty-seven eligible participants were randomly divided into 2 groups (real microcurrent group and sham microcurrent group) at a 1:1 allocation ratio. Outcome assessment and data analysis were performed by 2 physicians who were blinded to group allocation.

\section{Participants}

The subjects were patients over 18 years of age who had been diagnosed as chronic insomnia according to the International Classification of Sleep Disorders-Third Edition criteria. The patients with severe medical problems (severe cardiac, pulmonary, hepatic or renal problems) were initially excluded. And the patients who were being treated or had been treated with the psychiatric disorder were also excluded. Patients with following factors were additionally excluded; history of using medications that affects sleep 2 weeks before the study, hypersensitive reaction to ear-clip electrode, ineligibility as determined by a physician, refusal to participate in the trial or to provide informed consent, or an inability to comprehend or express oneself in the Korean language.

\section{Randomization}

Participants were randomized using a computerized random number generator by a professional statistician blinded to participant assignment in accordance with the Consolidated Standards of Reporting Trials statement. Block randomization was performed once the participants were confirmed to be eligible, and their written informed consent had been obtained. Participants were randomly assigned to the real microcurrent group or the sham microcurrent group. The partic- ipants, outcome assessors, and data analyst were masked to the allocation.

\section{Interventions}

We applied real or sham microcurrent electrical stimulation for 1 hour for 4 weeks. All treatment was given via electrodes clipped to the ear lobes, as illustrated in Fig. 1. The application points were established based on previous report. ${ }^{11}$ The microcurrent generator (Cosmic Co., Seoul, Korea) was a portable handheld device that provides output through 2 channels. It was programmed to provide an AC characterized by a monophasic rectangular pulse format, with polarity reversal every three seconds. The frequency was $8 \mathrm{~Hz}$ and the intensity was $25 \mu \mathrm{A}$. This level of current intensity was significantly below the human's threshold of sensation. The appearance of the sham microcurrent stimulator was identical to the real stimulator, but there was no electrical current, even when it was operational. All ear clip electrodes had a number marked on them, identifying each as real or sham treating, and the code was kept in a sealed envelope away from the study site.

The sleep hygiene education was performed separately for each participant at the time of enrollment. The following contents were instructed by the educated nurse with print materials and explanation; balanced eating habits, relaxing and

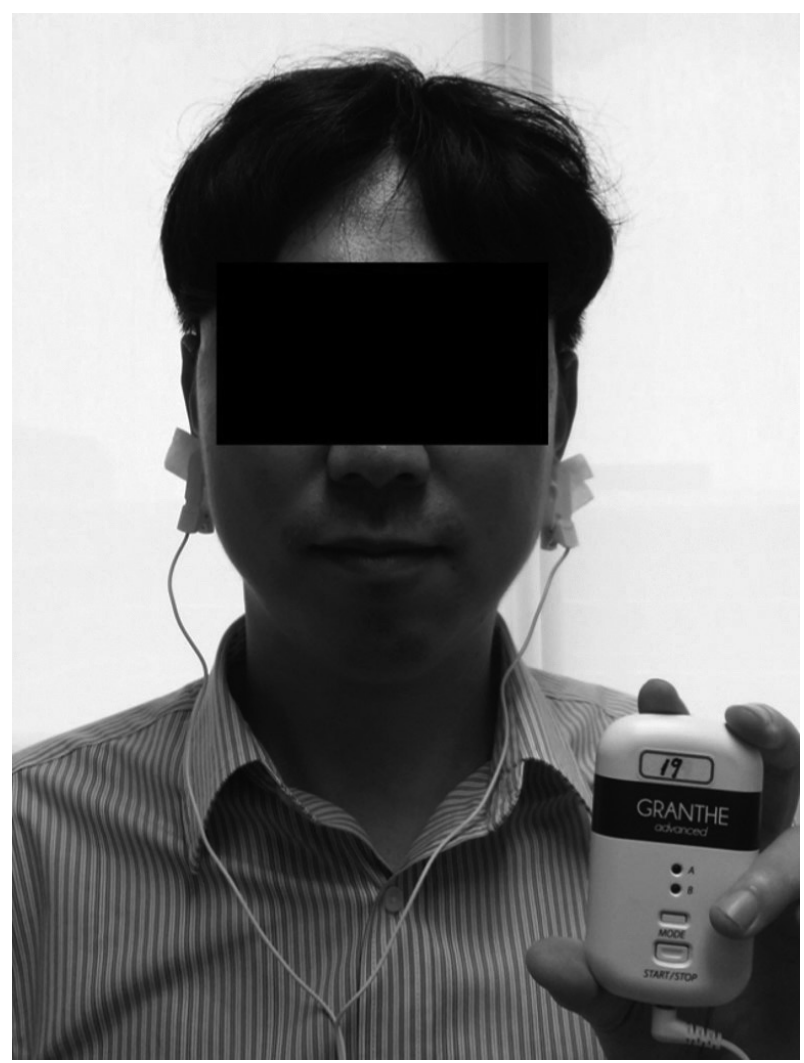

Figure 1. Ear clip electrode placement on patients in the study. 
comfort sleep environment, avoidance of substances (hypnotics, caffeine, alcohol and tobacco), avoidance of light exposure or television before bedtime, regular exercise. The participants, the staff, and the examining physician remained blind to the treatment conditions. After the study, the staff opened the code envelope and separated the patient evaluation forms into treatment and sham treatment. The statistician who evaluated the final study results remained blind to the treatment conditions.

\section{Outcome measurements}

All outcomes were measured at baseline (pre-treatment), 2 weeks post-treatment, and 4 weeks post-treatment. Body composition was measured at baseline and the details of adverse events were recorded throughout the trial.

\section{Pittsburgh Sleep Quality Index and Insomnia Severity Index}

The Pittsburgh Sleep Quality Index (PSQI) is a widely used tool for assessing subjective sleep quality. It is composed of 18 questions about quality, latency, duration, efficiency and disturbances of sleep, use of sleep medication, and daytime dysfunction. The questions are very easy to complete which takes 5 to 10 minutes and the score more than 5 suggests poor sleep quality. ${ }^{12}$ This study used the PSQI of Korean version which had been previously proved in reliability and validity. ${ }^{13}$

The Insomnia Severity Index (ISI) is a brief questionnaire reflecting the individual's perception about insomnia. It is composed of 7 questions assessing the difficulties of sleep onset and maintenance, satisfaction and concern about sleep pattern, difficulties in daytime function and noticeability of impairment in daily activity from sleep problems. The score from 7 to 14 suggests subthreshold insomnia and more than 15 suggests clinical insomnia. ${ }^{14}$ The study also used Korean version of ISI for outcome measurements. ${ }^{15}$

\section{Statistical analysis}

We estimated the sample size from the results of previous study. ${ }^{16}$ To detect this difference in a randomized controlled trial using an independent t-test with an alpha of 0.05 , a power of $80 \%$, and a $10 \%$ dropout rate, the suggested sample size was 15 subjects per group. Within-group changes were analyzed with a repeated measures analysis of variance. Intergroup differences were analyzed using the independent t-test. All variables with a $p<0.05$ were considered statistically significant. Statistical Package for the Social Sciences (SPSS) software 14.0 (SPSS Inc., Chicago, IL, USA) was used to conduct data analysis.

\section{Results}

A total of twenty-seven patients with age 44-78 years old participated in the study. The baseline PSQI and ISI score showed no statistically significant difference between the MC group and the sham group. The baseline characteristics of the participants are described in Table 1 . In sham group, the PSQI significantly decreased until 2 weeks of treatment $(p<0.01)$ (Table 2, Fig. 2). After 2 weeks of treatment, however, the PSQI increased. In MC group, the PSQI significantly decreased until 4 weeks of treatment $(p<0.01)$. The change of PSQI in MC group was smaller than that in sham group between baseline and 2 weeks of treatment $(p=0.018)$, and greater than that in sham group between 2 weeks and 4 weeks of treatment $(p=0.030$ ).

In sham group, the ISI significantly decreased until 2 weeks of treatment $(p<0.01)$. After 2 weeks of treatment, however, the ISI was not decreased. In MC group, the ISI significantly decreased until 4 weeks of treatment $(p<0.01)$. The change of ISI in MC group was greater than that in sham group be-

Table 1. Baseline characteristics

\begin{tabular}{lccc}
\hline \multirow{2}{*}{ Characteristics } & \multicolumn{2}{c}{ Group } & p-value* \\
\cline { 2 - 3 } & $\mathrm{MC}(\mathrm{n}=14)$ & Sham $(\mathrm{n}=13)$ & \\
\hline Female gender $(\mathrm{n})$ & $62 \pm 9$ & 11 & \\
Age & $24.5 \pm 2.6$ & $22.7 \pm 1.7$ & 0.04 \\
BMI $\left(\mathrm{kg} / \mathrm{m}^{2}\right)$ & $13.0 \pm 1.0$ & $13.0 \pm 2.0$ & 0.66 \\
Baseline PSQI & $21.0 \pm 3.0$ & $18.0 \pm 4.0$ & 0.40 \\
Baseline ISI & 9 & & 0.04
\end{tabular}

Values of age, BMI, baseline PSQI and baseline ISI are presented as mean \pm standard deviation. ${ }^{*}$ Independent t-test between MC and sham group. BMI: body mass index, PSQI: Pittsburgh Sleep Quality Index, ISI: Insomnia Severity Index, MC: cranial microcurrent therapy group, Sham: sham treatment group

Table 2. Comparison of changes in PSQI and ISI between MC and sham group

\begin{tabular}{lccllc}
\hline & \multicolumn{2}{c}{ PSQI } & & \multicolumn{2}{c}{ ISI } \\
\cline { 2 - 3 } \cline { 5 - 6 } & MC $(\mathrm{n}=14)$ & Sham $(\mathrm{n}=13)$ & & $\mathrm{MC}(\mathrm{n}=14)$ & Sham $(\mathrm{n}=13)$ \\
\hline Baseline & $12.7 \pm 1.5$ & $13.0 \pm 2.0$ & & $21.3 \pm 3.5$ & $18.2 \pm 4.5$ \\
$\Delta^{1}$ & $-1.6 \pm 2.2^{\dagger}$ & $-4.5 \pm 3.4^{\dagger}$ & & $-4.3 \pm 5.9$ & $-6.1 \pm 5.2$ \\
$2 \mathrm{WT}$ & $11.1 \pm 2.4^{*}$ & $8.5 \pm 3.6^{*}$ & & $17.0 \pm 5.2^{*}$ & $12.1 \pm 6.0^{*}$ \\
$\Delta^{2}$ & $-1.8 \pm 1.7^{\dagger}$ & $1.0 \pm 4.2^{\dagger}$ & & $-4.6 \pm 5.8^{\dagger}$ & $0.0 \pm 5.8^{\dagger}$ \\
$4 \mathrm{WT}$ & $9.3 \pm 2.9^{*}$ & $9.5 \pm 2.7^{*}$ & & $12.4 \pm 4.4^{*}$ & $12.1 \pm 5.7^{*}$ \\
\hline
\end{tabular}

Values are presented as mean \pm standard deviation. ${ }^{*} p<0.05$, derived from repeated measure of ANOVA for assessment time, ${ }^{\dagger} p<0.05$, derived from independent t-test between $\mathrm{MC}$ and sham group. PSQI: Pittsburgh Sleep Quality Index, ISI: Insomnia Severity Index, MC: cranial microcurrent therapy group, Sham: sham treatment group, $\Delta^{1}$ : difference between the value of baseline and 2WT, WT: week of treatment time, $\Delta^{2}$ : difference between the value of $2 \mathrm{WT}$ and $4 \mathrm{WT}$ 

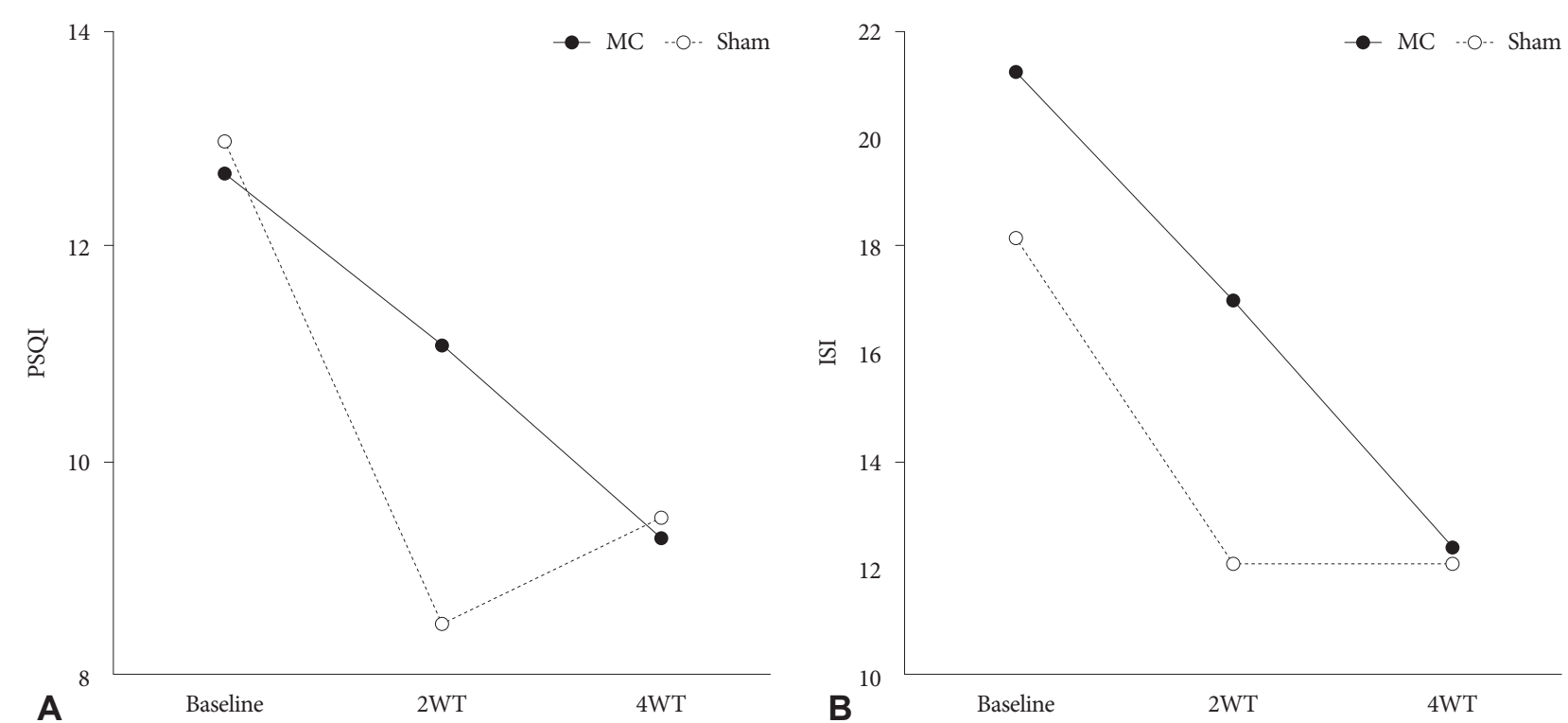

Figure 2. Sequential changes in PSQI and ISI at each week of treatment (A) PSQI (B) ISI. PSQI: Pittsburgh Sleep Quality Index, ISI: Insomnia Severity Index, WT: week of treatment time, MC: cranial microcurrent therapy group, Sham: sham treatment group.

tween 2 weeks and 4 weeks of treatment $(p=0.048)$.

\section{Discussion}

This is a randomized, double blinded and sham-controlled study to evaluate the effect of CES on subjective sleep quality in chronic insomnia patient. We found that CES-applied group showed consistently decreasing pattern of PSQI and ISI compared to that of sham group. The PSQI and ISI scores of both MC and sham group were decreased finally at 4 weeks compared to the baseline value. The difference between the two group was prominent in the period of 2-4 week. The PSQI and ISI scores of the MC group were still decreasing in the period of 2-4 week, while those of the sham group started increasing.

The mechanism how the CES made consistent effect for the MC group compared to sham group is not clearly understood. The evidence from the previous studies suggest that CES is somewhat effective for treating insomnia. A study by Lande and Gragnani demonstrated that CES was effective in increasing total sleep time. ${ }^{17}$ The study used a CES called "Alpha-stim" by Electromedical Products International, Inc. (Mineral Wells, TX, USA) for the military person with chronic insomnia. ${ }^{17}$ One of the early studies in 1970s was about investigating the effect of CES using electroencephalography. It revealed that CES treated group showed shortened sleep latency and the effect remained after 2 year of follow-up period. ${ }^{18}$

CES is useful not only in the aspect of effect but also in safety. The adverse effect of CES is reported to be less than $1 \%$ in human studies. The known adverse effects related to CES are vertigo, skin irritation due to electrodes, and headache. They are usually associated with too high currents for the individual and expected to subside by adjusting it. ${ }^{19}$ Vivid dream in patients with post-traumatic stress disorder is also reported. ${ }^{20}$ No adverse effects occurred in our study, which proves CES as a safe method.

Many of the previous studies were conducted with a specific population such as military person, cancer patients or severe depression. ${ }^{17,21,22}$ Our study excluded the patient with severe medical or psychiatric illness which made the effect of CES on sleep to be revealed more clearly.

Our study has several limitations. First, the short follow up period and small sample size. There was a change in tendency by 2 weeks in our results. A longer follow up period and larger sample size would have shown marked tendency of decrement or increment. Second, lack of consideration of objective sleep data. The endpoint of the study was the subjective sleep scales by individuals, not an objective data such as total sleep time or sleep latency. Considering these factors would make the future studies to have more level of completion.

In conclusion, this study suggests that a combination treatment of CES and sleep hygiene is more effective in treating chronic insomnia than a treatment only with sleep hygiene. We expect the CES to be an effective adjuvant therapy for treating chronic insomnia.

\section{Conflicts of Interest}

The authors have no potential conflicts of interest to disclose.

\section{Acknowledgments}

This research was supported by Basic Science Research Program through the National Research Foundation of Korea (NRF) funded by the 
Ministry of Education (NRF-2016R1D1A1B01014260).

\section{ORCID iDs}

Dong Rak Kwon Jung A Park Young Soo Lee Jae Hyuk Kwak Jin Kuk Do Ji Eun Kim

\section{Author Contributions}

Conceptualization: Dong Rak Kwon, Ji Eun Kim. Data curation: Ji Eun Kim, Jin Kuk Do, Young Soo Lee, Jae Hyuk Kwak. Formal analysis: Dong Rak Kwon. Funding acquisition: Dong Rak Kwon. Methodology: Dong Rak Kwon. Resources: Ji Eun Kim, Jin Kuk Do, Young Soo Lee. Supervision: Ji Eun Kim, Dong Rak Kwon. Validation: Dong Rak Kwon. Visualization: Dong Rak Kwon. Writing_original draft: Dong Rak Kwon, Ji Eun Kim, Jung A Park.

\section{REFERENCES}

1. Carter JR, Grimaldi D, Fonkoue IT, Medalie L, Mokhlesi B, Cauter EV. Assessment of sympathetic neural activity in chronic insomnia: evidence for elevated cardiovascular risk. Sleep 2018;41.

2. Javaheri S, Redline S. Insomnia and risk of cardiovascular disease. Chest 2017;152:435-444.

3. Morin CM, Bélanger L, LeBlanc M, et al. The natural history of insomnia: a population-based 3-year longitudinal study. Arch Intern Med 2009;169:447-453

4. Sateia MJ, Buysse DJ, Krystal AD, Neubauer DN, Heald JL. Clinical practice guideline for the pharmacologic treatment of chronic insomnia in adults: an American Academy of Sleep Medicine clinical practice guideline. J Clin Sleep Med 2017;13:307-349.

5. Matteson-Rusby SE, Pigeon WR, Gehrman P, Perlis ML. Why treat insomnia? Prim Care Companion J Clin Psychiatry 2010;12:PCC.08r00743.

6. Jonas WB. Cranial electrical stimulation: what is it, and should we use it in practice? Ann Intern Med 2018;168:446-447.

7. Zaghi S, Acar M, Hultgren B, Boggio PS, Fregni F. Noninvasive brain stimulation with low-intensity electrical currents: putative mechanisms of action for direct and alternating current stimulation. Neuroscientist 2010;16:285-307.

8. Lyon D, Kelly D, Walter J, Bear H, Thacker L, Elswick RK. Randomized sham controlled trial of cranial microcurrent stimulation for symptoms of depression, anxiety, pain, fatigue and sleep disturbances in women receiving chemotherapy for early-stage breast cancer. Springerplus 2015;4:369.

9. Kavirajan HC, Lueck K, Chuang K. Alternating current cranial electrotherapy stimulation (CES) for depression. Cochrane Database Syst Rev 2014;(7):CD010521.

10. Gilula MF, Kirsch DL. Cranial electrotherapy stimulation review: a safer alternative to psychopharmaceuticals in the treatment of depression. J Neurother 2005;9:7-26.

11. Lichtbroun AS, Raicer MM, Smith RB. The treatment of fibromyalgia with cranial electrotherapy stimulation. J Clin Rheumatol 2001;7:72-78.

12. Buysse DJ, Reynolds CF 3rd, Monk TH, Berman SR, Kupfer DJ. The Pittsburgh Sleep Quality Index: a new instrument for psychiatric practice and research. Psychiatry Res 1989;28:193-213.

13. Sohn SI, Kim DH, Lee MY, Cho YW. The reliability and validity of the Korean version of the Pittsburgh Sleep Quality Index. Sleep Breath 2012;16:803-812.

14. Bastien $\mathrm{CH}$, Vallières A, Morin CM. Validation of the Insomnia Severity Index as an outcome measure for insomnia research. Sleep Med 2001;2:297-307.

15. Cho YW, Song ML, Morin CM. Validation of a Korean version of the insomnia severity index. J Clin Neurol 2014;10:210-215.

16. Jacobs GD, Pace-Schott EF, Stickgold R, Otto MW. Cognitive behavior therapy and pharmacotherapy for insomnia: a randomized controlled trial and direct comparison. Arch Intern Med 2004;164:1888-1896.

17. Lande RG, Gragnani C. Efficacy of cranial electric stimulation for the treatment of insomnia: a randomized pilot study. Complement Ther Med 2013;21:8-13.

18. Weiss MF. The treatment of insomnia through the use of electrosleep: an EEG study. J Nerv Ment Dis 1973;157:108-120.

19. Kirsch DL, Nichols F. Cranial electrotherapy stimulation for treatment of anxiety, depression, and insomnia. Psychiatr Clin North Am 2013; 36:169-176.

20. Kirsch DL, Gilula MF. CES in the treatment of insomnia: a review and meta-analysis. Pract Pain Manag 2007;7:28-39.

21. Yennurajalingam S, Kang DH, Hwu WJ, et al. Cranial electrotherapy stimulation for the management of depression, anxiety, sleep disturbance, and pain in patients with advanced cancer: a preliminary study. J Pain Symptom Manage 2018;55:198-206.

22. Philip P, Demotes-Mainard J, Bourgeois M, Vincent JD. Efficiency of transcranial electrostimulation on anxiety and insomnia symptoms during a washout period in depressed patients. A double-blind study. Biol Psychiatry 1991;29:451-456. 